\title{
Identificación de los perfiles del tutor como resultado del funcionamiento de la acción tutorial
}

\author{
VÍCTOR León-CarrascosA* | María José FernándeZ-DíaZ**
}

La función tutorial favorece el desarrollo integral del individuo; pero para ello, los tutores deben presentar ciertas cualidades y competencias que derivan en diversas formas en su actividad educativa y tutorial. Esta investigación pretende identificar y analizar los perfiles de tutores atendiendo a la similitud de aspectos relativos a la planificación, las funciones del tutor, el desarrollo y la evaluación en la acción tutorial. Se trata de un estudio no experimental de carácter exploratorio. Se recoge información de un total de 401 tutores y 4,254 estudiantes de educación secundaria de la Comunidad de Madrid, España. Los resultados revelan cuatro perfiles de tutores con variedad de características en el desarrollo de sus funciones tutoriales. Se concluye que los centros tienen necesidad de establecer mecanismos de calidad para garantizar un buen funcionamiento de la acción tutorial a través del conocimiento de las realidades particulares y de los intereses de sus protagonistas.

Tutorial function favors the integral development of the individual; however, it is necessary that tutors present certain qualities and competencies which will, in consequence, derive in better educational and tutorial practices. The present research aims to identify and analyze the profiles of several tutors taking into account their similarities regarding such aspects as planning, functions, development and assessment. This is a non-experimental study of an exploratory nature. We gathered information from a total of 401 tutors and 4,254 secondary education students from Madrid, Spain. The results reveal four different tutor profiles with a variety of characteristics regarding the development of their tutorial functions. The conclusions reveal that educational centers need to establish quality mechanisms to guarantee the proper functioning of tutorial practices through the knowledge of the particular realities and interests of their students.
Palabras clave

Acción tutorial

Función tutorial

Perfil del tutor

Evaluación de la tutoría

Educación secundaria
Keywords

Tutorial practices

Tutorial function

Tutor profile

Tutorial assessment

Secondary education

Recepción: 19 de abril de 2020 | Aceptación: 25 de noviembre de 2020

DOI: https://doi.org/10.22201/iisue.24486167e.2021.174.59882

* Contratado doctor en el Centro Universitario (CES) Don Bosco adscrito a la Universidad Complutense de Madrid (UCM) (España). Doctor en Educación. Línea de investigación: innovación y calidad docente universitaria. Publicaciones recientes: (2019, en coautoría con M.J. Fernández-Díaz), "Diseño y validación de una escala para evaluar el funcionamiento de las tutorías en educación secundaria", Revista de Investigación Educativa (RIE), vol. 37, núm. 2, pp. 525-541. DOI: https://doi.org/10.6018/rie.37.2.345251; (2019, en coautoría con M.J. Fernández-Díaz), "Eficacia percibida en el desarrollo de las tutorías en educación secundaria”, Bordón. Revista de Pedagogía, vol. 71, núm. 2, pp.109-123. CE: victorleoncarrascosa@ucm.es

** Catedrática del Departamento de Investigación y Psicología en Educación del Centro de Formación del Profesorado de la Universidad Complutense de Madrid (UCM) (España). Líneas de investigación: metodología de investigación; evaluación y medida en educación; dirección y liderazgo; formación de profesores; calidad y evaluación de centros, profesores y programas educativos. Publicaciones recientes: (2019, en coautoría con J.M. RodríguezMantilla y V. León-Carrascosa), "Validation of a Questionnaire to Evaluate the Impact of ISO 9001 Standards in Schools with a Confirmatory Factor Analysis", Studies in Educational Evaluation, núm. 62, pp. 37-48; (2019, en coautoría con J.M. Rodríguez-Mantilla y F.J. Fernández-Cruz), "Comparative Analysis between Management Team and Teachers on the Impact of ISO 9001 Standards in Educational Centres", International Journal of Quality and Service Sciences, vol. 11, núm. 2, pp.248-264.CE: mjfdiaz@ucm.es 


\section{INTRODUCCIÓN}

El papel del tutor en la acción tutorial es fundamental a la hora de definir sus funciones. El tutor es una figura primordial en el desarrollo integral de los estudiantes. Cada centro educativo cuenta con variedad de profesionales que a través del ejercicio de las tutorías dan respuesta a la diversidad estudiantil; dicha respuesta depende, entre otros factores, del perfil de cada profesor tutor y de su implicación en el funcionamiento de la acción tutorial. Los tutores deben organizar la tutoría a través de criterios de sistematización, planificación y coherencia para que toda la comunidad educativa conozca de dónde parten, qué hacen y a qué responden sus acciones (Sanz, 2009). En este aspecto, el tutor y el resto del profesorado son las personas encargadas de hacer posible la acción tutorial en los centros educativos.

En la literatura encontramos numerosas acepciones del término tutor. Si bien, como señalan González-Benito y Vélaz de Medrano (2014), no hay un consenso en cuanto a su definición y sus funciones en el ámbito educativo, todas ellas se caracterizan por estar centradas en el desarrollo personal del estudiante. De las definiciones analizadas destacamos algunos rasgos y funciones que, de acuerdo con varios autores, se atribuyen al tutor (García Nieto, 2011; Cano González et al., 2013; Ley Orgánica para la Mejora de la Calidad Educativa, 2013; Yale, 2019):

- la responsabilidad de guiar y acompañar la evolución del estudiante;

- la coordinación de los procesos educativos de un grupo-clase atendiendo al desarrollo, maduración, orientación y aprendizaje del alumnado;

- el mantenimiento de la unión entre las diferentes personas implicadas en la educación de los estudiantes;

- el establecimiento de acciones continuas, programadas, integradas y colaborativas con el profesorado y las familias, en las que se tiene en cuenta el medio escolar, familiar y ambiental, donde se desarrollan habilidades, capacidades y competencias para el desarrollo integral;

- el empleo de diferentes recursos y estrategias a través de actividades de tipo funcional, orientador y formativo para que los estudiantes consigan lo mejor de sí mismos;

- y, finalmente, la intervención en todo el proceso que atañe al crecimiento del estudiante en el aula y con su grupo o clase.

Por otro lado, el perfil del tutor se caracteriza por incluir una actitud altruista, alta exigencia hacia el éxito, ganas de transformar la realidad educativa, compromiso y continuidad en su formación tutorial, así como una serie de cualidades y competencias que lo capacitan para establecer nexos con los demás. En este sentido, Giner (2012) recoge en uno de sus estudios la definición de competencia tutorial para el buen funcionamiento de las tutorías y el trabajo diario del tutor:

La capacidad de dar una respuesta lo más óptima posible, considerando el contexto y el entorno, a situaciones y problemas que se le plantean al profesorado-tutor en su ejercicio profesional, aplicando ciertas habilidades, conocimientos adecuados y tomando una actitud determinada, que está guiada por principios éticos (Giner, 2012: 28).

Otros autores hacen mención a la competencia docente como aquélla que integra los conocimientos, habilidades, motivos, valores y actitudes que un docente necesita para resolver con éxito las situaciones venideras en su quehacer profesional, y que le permiten acercarse a un ejercicio de calidad para dar soluciones eficientes a los problemas del día a día durante la acción educativa (Carbonero et al., 2010). Asimismo, las competencias del docente son parte de su desarrollo profesional, y se adquieren y mejoran de acuerdo con cada persona. 
El desempeño de la función del tutor debería ir ligada a ciertas actitudes relevantes, es decir, a una serie de cualidades para llevar a cabo de manera óptima las tareas que implica la acción tutorial. Diferentes estudios señalan, en opinión de los propios protagonistas, los docentes y los estudiantes, las cualidades más importantes que debe presentar un tutor (García Nieto, 2011; Jiménez y Navaridas, 2012; Youde, 2016):

Tabla 1. Cualidades del tutor (docentes-estudiantes)

\begin{tabular}{ll}
\hline \multicolumn{1}{c}{ Docentes } & \multicolumn{1}{c}{ Estudiantes } \\
\hline - Activo, sensible, flexible y con experiencia & - Un tutor cercano, sensible, alegre, simpático, afectuoso, \\
- Implicación y preocupación por los estudiantes & justo y amigable \\
- Rectitud y seriedad en las tareas y opiniones & Exigente, comprensivo y paciente, que transmita \\
- Constancia y paciencia & confianza \\
- Saber escuchar y dialogar & Una persona empática para que se sientan comprendi- \\
- Ser resolutivo y eficaz & dos y a gusto en clase \\
- "Saber hacerse niño, adolescente o joven, sin dejar de & Que conozca bien a sus tutelados y sea sincero y autén- \\
ser adulto" & tico con ellos \\
& $\begin{array}{l}\text { Con grandes habilidades sociales, que hable y dialogue } \\
\text { mucho con el grupo de tutelados }\end{array}$ \\
& Que sea afable y sepa orientar y aconsejar \\
& Que sea persistente y con iniciativa \\
& Que gestione conflictos con liderazgo \\
& Que realice actividades fuera del libro de texto adap- \\
& tadas al grupo de estudiantes (necesidades, intereses, \\
& motivaciones, etc.)
\end{tabular}

Fuente: elaboración propia.

A su vez, dichas cualidades van ligadas a las competencias básicas del tutor. Vélaz de Medrano et al. (2013) determinan un perfil educativo ligado al conocimiento y desempeño de las funciones tutoriales que éste atiende: la orientación personal, académica, familiar, profesional; la evaluación psicopedagógica, la dinamización de la tutoría, el asesoramiento y colaboración con el profesorado en las dificultades del proceso de enseñanza-aprendizaje; la innovación y mejora de la calidad de la educación, la resolución de conflictos y la coordinación de equipos.

También son parte de las tareas del tutor la planificación, impartición, tutorización y evaluación de las acciones formativas. Waltz (2019) aporta una serie de competencias que permiten dar respuesta a la atención de los estudiantes: una relación cercana con el alumnado, ser experto en el ámbito de conocimientos, facilitador de aprendizajes, diseñador de medios y recursos didácticos, promotor de la calidad de la formación y actualizador didáctico; orientador y motivador, evaluador, cooperador con el resto del profesorado, profesional reflexivo respecto de sus acciones y abierto a las innovaciones. En suma, Carbonero et al. (2010) indican dos competencias que se complementan mutuamente: la competencia científico-didáctica y la personal-instrumental. Y las presentan en tres bloques de acuerdo con el lugar en el que adquieren mayor protagonismo: 1) la autoeficacia, la planificación y la toma de decisiones; 2) la comunicación, resolución de conflictos (mediación), vinculación afectiva, empatía, asertividad, liderazgo y convivencia; y 3) la adaptación a nuevas situaciones. Asimismo, González-Lorente y González-Morga (2015) se centran en el modelo de competencia de acción profesional (CAP) y describen las herramientas adecuadas que un tutor debe de tener: las competencias técnicas 
/ saber, referidas al conjunto de conocimientos psicopedagógicos que sustenta la base formativa para su guía en la acción tutorial; las competencias metodológicas / saber hacer, que son las competencias técnicas con la capacidad de ponerlas en práctica durante el desarrollo de la acción tutorial; las competencias participativas / saber estar, referidas a la habilidad para comunicarse y mantener una actitud abierta a la comprensión de distintos puntos de vista; y la competencia personal / saber ser, que se refiere a la unión de una serie de cualidades propias de la personalidad y compromiso ético y social del tutor, con clara dedicación a ayudar, mediar, orientar y redirigir a las personas en sus proyectos personales y de vida.

Según Carvalho y Taveira (2014) los tutores influyen directamente a través de las relaciones que establecen en el aula y la asignatura que enseñan, y obtienen información de los estudiantes relativa a asuntos escolares, personales y profesionales. En este sentido, Bereziartua et al. (2017) destacan resultados importantes sobre el papel de la acción tutorial en los momentos de tránsito entre etapas educativas. Concretamente, la tutoría debería incluir acciones de aproximación al final de cada etapa, desarrollar acciones puntuales al comienzo del nuevo curso y acciones de asentamiento y seguimiento del periodo de adaptación para asegurar la integración en la vida escolar.

Arnaiz et al. (2015) identifican diferentes barreras que se deben de tener en cuenta en los planes de mejora de la educación inclusiva, en alusión a mejoras en la organización de la acción tutorial relacionada con los procesos de aprendizaje. Igualmente, Castellano y Pantoja (2017) señalan el valor del uso de las tecnologías para apoyar el proceso de aprendizaje en el sentido de lograr una mejor relación con el alumnado y su familia. Por otro lado, Marcelo y Yot (2013) destacan cómo la función tutorial está en constante evolución, dada la incorporación de herramientas y recursos tecnológicos que desafían continuamente las tareas educativas y tutoriales de los docentes.
Si bien existen diversos estudios centrados en determinar el perfil profesional de los tutores en los que se aprecian coincidencias en torno a las funciones tutoriales (Jiménez y Navaridas, 2012; Vélaz de Medrano et al., 2013; Youde, 2016; Delgado-García, et al., 2020), el presente trabajo aporta mayor información a la práctica tutorial en la medida en que toma en cuenta otros componentes implicados en el funcionamiento de la acción tutorial, como la planificación, las funciones del tutor, el desarrollo de su actividad y la evaluación. De acuerdo con la literatura científica, estos cuatro componentes figuran entre las cualidades y competencias del tutor en el modo de desempeñar sus tareas tutoriales en los centros educativos, y se justifican a continuación.

- La planificación de la acción tutorial se basa en los procesos organizativos de la práctica de la tutoría. La tutoría debe responder a las necesidades y características individuales de sus protagonistas con la intención de favorecer su desarrollo integral (Cruz, 2018). Todo ello debe materializarse en un documento donde se recoge la organización, gestión y toma de decisiones, ya que se trata de una acción planificada y no de algo improvisado y aislado; de esta manera se otorga mayor reconocimiento a los procesos formativos desde la práctica docente (Martínez et al., 2018).

- Las funciones del tutor determinan las áreas de intervención durante el desarrollo de las tutorías, y son de tres tipos: con el alumnado, con la familia y con el profesorado. El tutor representa una figura clave en las relaciones con el grupo-clase y los procesos de enseñanza, aprendizaje y formativos aplicados dentro de los escenarios educativos desde los diferentes ámbitos de la vida (social, personal, escolar o profesional) (Alegre et al., 2017; López y González, 2018). Por otro lado, las intervenciones 
con la familia están destinadas a mejorar su participación para contribuir al progreso educativo de sus hijos (Álvarez-Justel, 2017). Por último, la colaboración con el resto del profesorado es un valor añadido en la práctica tutorial, ya que la respuesta a las necesidades de los estudiantes mejora con el desarrollo de actividades consensuadas y focalizadas entre todo el profesorado que interviene con el alumnado (Urosa y Lázaro, 2017).

- El desarrollo de las tutorías se centra en el empleo de programas educativos en relación con la realidad social y las necesidades de los estudiantes. En la literatura científica se observa un mayor predominio de programas educativos que permiten acercar el trabajo de la acción tutorial con la actualidad (Bejar, 2018; Sánchez-Porro y González Ortega, 2017); sin embargo, también existen programas centrados en el desarrollo de valores, emociones y habilidades para la vida que son fundamentales para el progreso personal de los estudiantes (LeónCarrascosa y Fernández-Díaz, 2019b; Martínez et al., 2019).

- La evaluación de las tutorías sintetiza su funcionamiento. Se trata de una herramienta que mantiene activa la acción tutorial desde un planteamiento de mejora continua. Para ello, se obtiene información de los resultados satisfactorios, se refuerzan los aspectos a mejorar y se modifican las acciones puestas en marcha con la finalidad de lograr una mayor efectividad, eficacia y funcionalidad (Pérez et al., 2017; Ponce et al., 2020). El tutor se caracteriza por su escucha activa y la retroalimentación llevada a cabo en todo el proceso evaluador (De la Torre-Laso, 2019; Torquemada y Jardínez, 2019).

A pesar de las particularidades expuestas sobre el funcionamiento de las tutorías en los centros educativos, destaca que hay pocos estudios centrados en la identificación de perfiles que revelen la dedicación del profesorado tutor y permitan analizar las características de cada uno a la hora de desempeñar su trabajo.

Ante esta realidad -y la falta de concreción de un perfil del tutor centrado en el modo de proceder en la acción tutorial- el presente trabajo tiene como objetivo principal identificar y analizar los perfiles de tutores respecto de los aspectos más comunes referidos a la planificación, las funciones del tutor, el desarrollo y la evaluación de la acción tutorial, tomando en cuenta, en un caso, las valoraciones de los propios tutores y, en otro, las de los estudiantes tutorados.

\section{MÉtodo}

\section{Participantes}

La población de nuestro estudio está conformada por profesores tutores de educación primaria y educación secundaria obligatoria y postobligatoria de los centros públicos, privados y concertados de las cinco Direcciones de Área Territoriales (DAT) de la Comunidad de Madrid (CM), España; y, por otro lado, por los estudiantes de educación secundaria obligatoria y postobligatoria de la CM. El tipo de muestreo utilizado fue incidental; la muestra fue de 401 tutores y 4 mil 254 estudiantes.

De los tutores, 56.1 por ciento pertenece a centros públicos, 30.4 por ciento a centros concertados y 13.5 por ciento a centros privados. La distribución por zonas territoriales del profesorado muestra que 5.2 por ciento pertenece a la zona norte, 16.2 por ciento a la zona sur, 5.5 por ciento a la zona este, 20 por ciento a la zona oeste y 53.1 por ciento a la zona centro. Los datos sociodemográficos están formados por 68.3 por ciento mujeres y 31.7 por ciento hombres, de los cuales 29.4 por ciento presentan un rango de edad de 35 años o menos, 37.9 por ciento entre 36 y 45 años y 42.7 por ciento tienen 46 años o más. Asimismo, desde el punto de vista profesional, 18.2 por ciento 
cuenta con 5 años o menos de experiencia docente, 20.9 por ciento entre 6 y10 años y 60.8 por ciento más de 11 años. Por otro lado, 31.4 por ciento manifiesta que tienen 5 años o menos de experiencia como tutor, 29.2 por ciento entre 6 y 10 años y 39.4 por ciento tiene 11 años o más.

En cuanto a los estudiantes, 32.1 por ciento pertenece a centros públicos, 44.9 por ciento a centros concertados y 23 por ciento a centros privados. La distribución por zonas refleja que 4.4 por ciento pertenecen a la zona norte, 2.3 por ciento a la zona sur, 26.9 por ciento a la zona oeste y 66.4 por ciento a la zona centro. La muestra no cuenta con estudiantes de la zona este. Atendiendo a los datos sociodemográficos, 50 por ciento son mujeres y la misma proporción, hombres. Respecto al rango de edad, 54.2 por ciento tiene 14 años o menos, 32.8 por ciento entre $15-16$ años y 13 por ciento tiene 17 años o más. En cuanto al número de tutorías que se imparten en el centro, 91.7 por ciento de los estudiantes indica que éstas se imparten una vez a la semana y la forma de participación más común (91.1 por ciento) es en el grupo-clase.

\section{Instrumento}

Para la identificación de los diferentes perfiles de tutores en los centros educativos se utilizaron dos instrumentos de medida en relación con cada una de las muestras participantes. Dichos instrumentos fueron de elaboración propia, fundamentados teóricamente a partir de la literatura científica y de nuestra experiencia sobre el objeto de estudio.

El cuestionario destinado a los tutores sobre el funcionamiento de la acción tutorial considera cuatro dimensiones que configuran un total de 92 ítems: planificación de la acción tutorial (atiende el estudio de la realidad y la organización de las tutorías); funciones del tutor (comprende el tipo de actuaciones con los alumnos, las familias y el profesorado); desarrollo de la acción tutorial (indica los programas y actividades tutoriales, las acciones realizadas en el aula y el empleo de recursos interactivos); y evaluación de la acción tutorial (vinculada al resto de las dimensiones en relación con el seguimiento, la recogida de información y los resultados). Cada ítem del instrumento se valoró en una escala tipo Likert de 1 a 5, donde 1 indica nada y 5 indica mucho. La fiabilidad del instrumento fue excelente, ya que alcanzó un alfa de Cronbach de 0.968 (León-Carrascosa, 2018).

El cuestionario destinado a los estudiantes sobre la valoración de las tutorías cuenta, al igual que el cuestionario para profesores, con cuatro dimensiones centradas en aquellos aspectos que los estudiantes observan desde el trabajo de la tutoría en el aula, y está configurado con un total de 29 ítems: funciones del tutor con el alumnado (comprende la capacidad de resolución de problemas y las competencias del tutor en el aula); funciones del tutor con las familias (entendidas como aquellas acciones que el estudiante percibe en las relaciones familia-escuela); desarrollo de las tutorías (abarca las actividades y la orientación recibida por parte del tutor); y evaluación de las tutorías (alude a la valoración personal de los estudiantes sobre las tutorías). De igual modo que en la escala anterior, en este caso el estudiante debe responder a los ítems con opciones de respuesta de 1 a 5 (escala tipo Likert). La fiabilidad de este instrumento también fue excelente, con un alfa de Cronbach de 0.929 (León-Carrascosa y Fernández-Díaz, 2019a).

\section{Diseño y procedimiento}

El diseño implicado en este estudio es no experimental de carácter exploratorio. La recogida de información se realizó a lo largo del curso académico 2016-2017 de manera intencionada con la finalidad de contrastar su funcionamiento como una tarea implícita dentro de las funciones de los docentes en los centros educativos. El proceso seguido consistió en contactar con los centros educativos de educación primaria y secundaria de la Comunidad de Madrid a través del correo electrónico que se encuentra en la base de datos de 
la Consejería de Educación. En dicho correo se explicaba el objeto de estudio y la solicitud de colaboración en el mismo. A quienes aceptaron participar se les facilitó el número de ejemplares de cuestionarios según la demanda de cada centro, con un margen de 2-3 semanas para su recogida; se garantizó el anonimato y confidencialidad de la información obtenida. A los centros que participaron se les entregó, como agradecimiento, un informe final individualizado con los resultados para la mejora de la práctica tutorial en el centro.

\section{Análisis de datos}

Para el análisis de datos se realizó un estudio inicial de las medias, desviaciones típicas y frecuencias en las respuestas de ambas muestras, y se observó que la tendencia de las respuestas del profesorado era más alta (media más alta 4.76 y desviación típica 0.552: ítem 38), teniendo en cuenta el factor de autocomplacencia, mientras que los estudiantes contaban con valores más heterogéneos (media más alta 4.273 y desviación típica 1.472: ítem 5). Con el fin de identificar los diferentes perfiles del profesorado en relación con el funcionamiento de la acción tutorial y la valoración de las tutorías se utilizó el programa informático estadístico SPSS versión 22 (Statistical Package for the Social Sciences), concretamente, la técnica de análisis cluster, la cual parte de un conjunto de datos inicialmente no clasificados, en el que se analiza la distancia entre los elementos a través de un proceso iterativo hasta que cada elemento se asigna a un grupo. Asimismo, dentro del análisis cluster se utilizó el método K-medias, a partir del número de cluster fijado, ya que si el número es demasiado elevado podemos encontrar problemas de interpretación, y si el número es demasiado bajo, esto puede ser resultante de una inadecuada representación de la muestra de estudio en los clusters.

\section{Resultados}

\section{Percepción de los tutores}

Para obtener los resultados sobre los perfiles de tutores en las respuestas de los profesores tutores participantes se utilizó el método K-medias, y se tomaron como criterio de análisis las categorías establecidas en cada variable de 1 a 5 (1=nada/casi nada; $5=$ totalmente/ mucho) para el establecimiento de los conglomerados. El primer análisis cluster, fijado a 5 conglomerados, mostró los siguientes resultados: se observó que apenas existían diferencias entre los conglomerados 1 y 3 , y los conglomerados 2 y 5; asimismo, se observó que las variables 2, 3, 21, 22, 25, 37, 63, 64, 65, 66 y 67 presentaban una baja variabilidad en las puntuaciones correspondientes a los distintos conglomerados, por lo que resultaban poco discriminantes, y por ello fueron eliminadas.

Ante estos resultados, se tomaron dos decisiones: se modificó el número de conglomerados a 3, pero se llevó a cabo un análisis cluster fijado a 4 conglomerados para ver el comportamiento de las variables, con lo que se encontraron dos conglomerados casi sin diferencias y se eliminaron las variables mencionadas anteriormente (según indicaciones de Hair et al., 2009).

De este modo, al realizar el análisis se obtuvieron resultados adecuados y de sencilla y significativa interpretación. En la Tabla 2 se muestran los resultados de los centros de conglomerados finales en los que se estudiaron los posibles perfiles identificados de la figura del tutor en el funcionamiento de la acción tutorial por parte del profesorado. 


\section{Tabla 2. Centro de conglomerados finales (profesorado tutor)}

Método K-medias. Solución 3 conglomerados

\begin{tabular}{|c|c|c|c|}
\hline Ítems & \multicolumn{3}{|c|}{ Conglomerado } \\
\hline \multicolumn{4}{|l|}{ Planificación } \\
\hline 1. En general, en el centro se realizan estudios de necesidades del alumnado & 3.75 & 4.38 & 3.29 \\
\hline 4. En nuestro centro la acción tutorial se ve favorecida por una buena relación con las familias & 3.86 & 4.29 & 3.40 \\
\hline 5. Realizo las actividades de tutorías en colaboración con el Departamento de Orientación & 3.90 & 4.52 & 3.08 \\
\hline $\begin{array}{l}\text { 6. El plan de acción tutorial (PAT) está elaborado en coherencia con el resto de los documentos } \\
\text { organizativos }\end{array}$ & 4.16 & 4.63 & 3.49 \\
\hline 7. El profesorado del centro considera prioritario el desarrollo de la labor tutorial & 4.01 & 4.39 & 3.45 \\
\hline 8. El PAT está elaborado a partir de las aportaciones del curso anterior & 3.90 & 4.47 & 3.03 \\
\hline 9. Se planifican pautas para llevar a cabo la comunicación y participación de las familias & 3.80 & 4.23 & 3.23 \\
\hline 10. Sigo las líneas de actuación establecidas en el PAT & 4.03 & 4.53 & 3.46 \\
\hline 11. Los tutores organizan y programan las actividades a realizar durante el curso & 3.84 & 4.31 & 3.45 \\
\hline $\begin{array}{l}\text { 12. Se establecen niveles y ámbitos de intervención según la prioridad y el grado de necesidad } \\
\text { que requiera el grupo-clase }\end{array}$ & 3.90 & 4.41 & 3.05 \\
\hline $\begin{array}{l}\text { 13. La mayoría de los tutores tenemos formación suficiente para el ejercicio de la acción } \\
\text { tutorial }\end{array}$ & 3.71 & 4.20 & 3.19 \\
\hline 14. Conozco las funciones de cada profesional en el desarrollo de la acción tutorial & 3.87 & 4.37 & 3.27 \\
\hline 15. Los tutores se implican activamente en la planificación de las tutorías & 4.15 & 4.47 & 3.75 \\
\hline 16. Los tutores aportamos nuevos contenidos o temáticas para trabajar en las tutorías & 3.96 & 4.28 & 3.31 \\
\hline $\begin{array}{l}\text { 17. Los tutores aprovechamos el entorno natural y urbano para que la tutoría esté más conecta- } \\
\text { da con la realidad }\end{array}$ & 2.65 & 3.24 & 2.35 \\
\hline 18. Participo en la elaboración del PAT & 3.09 & 3.85 & 2.68 \\
\hline \multicolumn{4}{|l|}{ Funciones del tutor } \\
\hline 19. Los tutores conocemos la situación familiar de nuestros alumnos y sus problemas & 4.20 & 4.45 & 3.75 \\
\hline 20. Conozco bien a cada alumno & 4.20 & 4.53 & 3.94 \\
\hline $\begin{array}{l}\text { 23. Las actividades que trabajo en las tutorías son dedicadas a resolver aquellos problemas que } \\
\text { surgen en el aula }\end{array}$ & 4.10 & 4.53 & 3.42 \\
\hline $\begin{array}{l}\text { 24. Promuevo el trabajo en equipo para favorecer la comunicación, la cohesión del grupo y } \\
\text { abordar posibles problemas de integración }\end{array}$ & 4.38 & 4.73 & 3.84 \\
\hline 26. Los alumnos participan en el desarrollo de las normas de comportamiento de su aula & 4.04 & 4.42 & 3.47 \\
\hline 27. Establezco el reparto de tareas, cargos y roles de los alumnos en el aula & 4.22 & 4.62 & 3.83 \\
\hline 28. Potencio el desarrollo de hábitos básicos de autonomía personal y social & 4.29 & 4.67 & 3.90 \\
\hline 29. Informo a los padres sobre los objetivos individuales propuestos para su hijo & 4.18 & 4.73 & 3.77 \\
\hline $\begin{array}{l}\text { 30. Informo periódicamente a la familia sobre la conducta y el rendimiento académico del } \\
\text { alumno }\end{array}$ & 4.33 & 4.74 & 3.85 \\
\hline $\begin{array}{l}\text { 31. Informo a las familias sobre la normativa educativa, la organización y el funcionamiento } \\
\text { del centro }\end{array}$ & 4.27 & 4.74 & 3.86 \\
\hline $\begin{array}{l}\text { 32. Oriento a los padres sobre la forma de abordar los posibles problemas según el momento } \\
\text { evolutivo del alumno }\end{array}$ & 4.27 & 4.71 & 3.69 \\
\hline 33. Mantengo informadas a las familias sobre posibles servicios de apoyo & 4.23 & 4.67 & 3.57 \\
\hline 34. Solicito la colaboración y participación de los padres en la vida del centro & 3.77 & 4.26 & 3.38 \\
\hline $\begin{array}{l}\text { 35. Tenemos en cuenta las propuestas de las familias para su información y participación en el } \\
\text { centro }\end{array}$ & 3.76 & 4.33 & 3.39 \\
\hline
\end{tabular}




\section{Tabla 2. Centro de conglomerados finales (profesorado tutor)}

Método K-medias. Solución 3 conglomerados

\section{Ítems}

Conglomerado

36. Realizamos reuniones con el grupo de familias para informar y analizar sobre el desarrollo $\begin{array}{llll}4.12 & 4.73 & 3.82\end{array}$ del curso

38. Utilizo información de cursos anteriores que han aportado otros tutores para el desarrollo $\quad \begin{array}{llll}3.97 & 4.54 & 3.43\end{array}$ de mi acción tutorial

\begin{tabular}{|c|c|c|c|}
\hline Analizo con los profesores las dificultades de los alumnos para abordarlas & 4.24 & 4.73 & 3.62 \\
\hline 40. Me coordino con el resto de profesores que imparten docencia con el grupo clase & 4.16 & 4.74 & 3.68 \\
\hline $\begin{array}{l}\text { 41. Los tutores realizamos reuniones con el equipo docente de nuestros grupos de alumnos } \\
\text { para coordinar criterios, ajustes en las programaciones, medidas educativas o de refuerzo, etc. }\end{array}$ & 3.87 & 4.60 & 3.05 \\
\hline $\begin{array}{l}\text { 42. Los tutores realizamos periódicamente reuniones con los demás tutores de los diferentes } \\
\text { cursos, ciclos o niveles }\end{array}$ & 3.73 & 4.42 & 2.48 \\
\hline $\begin{array}{l}\text { 43. El jefe de estudios mantiene reuniones periódicas con los tutores para coordinar el buen } \\
\text { funcionamiento de la acción tutorial }\end{array}$ & 3.66 & 4.37 & 2.89 \\
\hline 44. Recurro a profesores del centro para tratar temas específicos de interés en las tutorías & 3.12 & 4.09 & 2.62 \\
\hline $\begin{array}{l}\text { 45. Contamos con otros profesionales externos para tratar aquellos temas poco comunes y } \\
\text { menos conocidos }\end{array}$ & 3.46 & 4.30 & 2.57 \\
\hline 46. Los tutores contamos con el apoyo del centro para el desarrollo de las tutorías & 4.23 & 4.70 & 3.28 \\
\hline 47. El profesorado dispone de tiempo para organizar las tutorías con los alumnos & 2.22 & 3.26 & 1.85 \\
\hline 48. En general, los tutores disponemos de espacios adecuados para la $\mathrm{r}$ & 3.54 & 4.30 & 2.75 \\
\hline
\end{tabular}

\section{Desarrollo}

49. Desarrollo programas de habilidades sociales en el centro educativo y en el aula

$3.87 \quad 4.31 \quad 3.24$

50. Llevo a cabo diferentes técnicas y estrategias para el desarrollo del pensamiento crítico y $\begin{array}{llll}4.06 & 4.51 & 3.48\end{array}$ constructivo

51. El alumno está asesorado en el empleo de su tiempo y actividades fuera del contexto

$3.54 \quad 4.26 \quad 2.72$
escolar

52. Trabajamos con programas para el desarrollo de estilos de vida saludable

$\begin{array}{lll}3.83 & 4.49 & 3.20\end{array}$

53. Realizamos programas de orientación y apoyo al aprendizaje, previniendo las dificultades $\quad \begin{array}{llll}3.73 & 4.53 & 3.03\end{array}$ que puedan surgir y las medidas a adoptar

54. Aplicamos programas para ayudar a los alumnos en la construcción de su identidad personal

$3.52 \quad 4.29 \quad 2.98$

55. Aplicamos programas para mejorar en los alumnos la capacidad de aprender y de pensar

56. Aplicamos programas para que aprendan a tomar decisiones realistas y responsables sobre $\quad \begin{array}{llll}3.58 & 4.40 & 2.94\end{array}$ su futuro

\begin{tabular}{llcc}
$\begin{array}{l}\text { 57. Oriento sobre estrategias de aprendizaje y técnicas de estudio para el desarrollo de las } \\
\text { materias }\end{array}$ & 3.98 & 4.56 & 3.47 \\
\hline 58. Concienciamos a los alumnos sobre la igualdad de género en todos los ámbitos de la vida & 4.40 & 4.83 & 3.80 \\
\hline $\begin{array}{l}\text { 59. Proporciono a los alumnos información sobre las opciones académicas y profesionales } \\
\text { 60. Presento a los alumnos el programa de tutorías y les invito a proponer actividades }\end{array}$ & 3.38 & 4.13 & 2.85 \\
\hline 61. Realizo dinámicas de grupo para evaluar la acción tutorial & 3.89 & 2.40 \\
\hline 62. Realizo debates sobre temas de actualidad que son de interés en la sociedad & 3.40 & 4.10 & 2.46 \\
\hline $\begin{array}{l}\text { 68. Utilizo documentos, libros, periódicos... de carácter formativo y materiales prácticos } \\
\text { (manipulables) en las tutorías }\end{array}$ & 3.32 & 4.13 & 2.76 \\
\hline $\begin{array}{l}\text { 69. Registro la información durante las reuniones, entrevistas y actividades desarrolladas } \\
\text { durante las tutorías }\end{array}$ & 3.95 & 2.10 \\
\hline
\end{tabular}




\begin{tabular}{|c|c|c|c|}
\hline \multirow{2}{*}{\begin{tabular}{l}
\multicolumn{1}{c}{ Ítems } \\
$\begin{array}{l}\text { 70. Dispongo del material necesario para cumplir con mi tutoría: listas, teléfonos, impresos de } \\
\text { comunicación, etc. }\end{array}$
\end{tabular}} & \multicolumn{3}{|c|}{ Conglomerado } \\
\hline & 4.14 & 4.72 & 3.48 \\
\hline 71. Utilizo medios audiovisuales para realizar las tutorías & 3.66 & 4.49 & 2.51 \\
\hline $\begin{array}{l}\text { 72. Utilizo las tecnologías de la información y la comunicación para el desarrollo de la acción } \\
\text { tutorial }\end{array}$ & 3.77 & 4.42 & 2.67 \\
\hline 73. Facilito la interacción virtual, tanto con el alumnado como con las familias & 3.15 & 3.94 & 2.37 \\
\hline 74. Trabajo la tutoría con algunas herramientas que dispone la red informática/web & 2.96 & 3.72 & 1.96 \\
\hline $\begin{array}{l}\text { 75.- El centro cuenta con una plataforma interactiva de acceso privado para la información y } \\
\text { contacto con la familia }\end{array}$ & 3.45 & 4.21 & 2.42 \\
\hline \multicolumn{4}{|l|}{ Evaluación } \\
\hline 76. Se cuenta con un cronograma del desarrollo de la acción tutorial en el centro & 3.20 & 4.05 & 2.26 \\
\hline 77. Existe un programa de actividades concreto dentro del PAT & 3.88 & 4.46 & 2.90 \\
\hline 78. Los tutores destinamos suficiente tiempo a las tutorías con los padres & 3.83 & 4.40 & 3.16 \\
\hline $\begin{array}{l}\text { 79. Sigo un programa de tutoría reglado por organismos estatales, disponibles en la literatura } \\
\text { u otros }\end{array}$ & 1.99 & 2.98 & 1.59 \\
\hline $\begin{array}{l}\text { 80. El centro educativo tiene los recursos económicos suficientes para facilitar material para la } \\
\text { tutoría }\end{array}$ & 3.32 & 4.10 & 2.63 \\
\hline 81. La administración educativa facilita recursos para la realización de las tutorías en el centro & 2.59 & 3.54 & 1.76 \\
\hline 82. Se realizan modificaciones durante el curso en el PAT & 3.80 & 4.58 & 2.82 \\
\hline 83. Se actualizan los contenidos de las tutorías durante el curso & 3.80 & 4.51 & 2.78 \\
\hline $\begin{array}{l}\text { 84. El nivel de participación de la comunidad educativa es suficiente para el desarrollo de las } \\
\text { tutorías }\end{array}$ & 3.59 & 4.16 & 2.90 \\
\hline 85. Se aplican encuestas para valorar la práctica tutorial & 2.55 & 3.60 & 1.72 \\
\hline 86. Se recoge información sobre el grado de satisfacción de los alumnos & 2.80 & 3.97 & 1.98 \\
\hline 87. Los tutores realizamos autoevaluaciones de las sesiones de tutoría & 2.86 & 3.94 & 2.04 \\
\hline 88. Los tutores estamos satisfechos con las tareas realizadas en las tutorías & 3.47 & 4.19 & 2.62 \\
\hline 89. Se proponen acciones de mejora a partir de la detección de los puntos fuertes y débiles del PAT & 3.51 & 4.32 & 2.49 \\
\hline $\begin{array}{l}\text { 90. Se realiza una evaluación final del trabajo realizado con el fin de plantear los objetivos para } \\
\text { el siguiente curso }\end{array}$ & 3.65 & 4.39 & 2.76 \\
\hline $\begin{array}{l}\text { 91. En general, observo continuidad en el desarrollo de la acción tutorial en el alumnado hasta } \\
\text { finalizar su educación obligatoria }\end{array}$ & 3.47 & 4.32 & 2.59 \\
\hline 92. La comunidad educativa valora positivamente el PAT & 3.64 & 4.44 & 2.77 \\
\hline
\end{tabular}

Fuente: elaboración propia.

Tras las modificaciones realizadas, los resultados con tres conglomeraciones fueron satisfactorios y se pudo definir cada uno de los cluster con mayor exactitud: conglomerado 1: tutor con dedicación alta; conglomerado 2: tutor con dedicación moderada; y conglomerado 3: tutor con dedicación leve.

Para proceder a la descripción de los perfiles, seguimos las indicaciones de Hair et al.
(2009), quienes señalan la conveniencia de asignar, a cada conglomerado, una etiqueta que especifique la naturaleza de cada uno y el análisis de las medias obtenidas por los elementos de los conglomerados (ya sea a través del análisis discriminante o del análisis de varianza). En nuestro caso, optamos por el análisis de varianza. En primer lugar, se realizó un estudio previo para conocer si se cumple o no la homogeneidad de 
varianzas a través de la prueba de Levene, con el fin de aplicar los contrastes posteriores a través del estadístico de Game-Howell (en el caso de incumplimiento de homogeneidad) y el estadístico de Scheffé (para los demás casos).

$\mathrm{Al}$ analizar los estudios posteriores se observó que todas las dimensiones presentan diferencias significativas entre todos los grupos, y que se cumplen los siguientes criterios: el conglomerado 1 presenta diferencias entre las medias más altas respecto al conglomerado 3, y más moderadas respecto del conglomerado 2; asimismo, el conglomerado 2 presenta puntuaciones más altas respecto del conglomerado 3. Por ello, a la hora de exponer los resultados nos limitaremos a destacar las diferencias significativas en las variables que observamos más relevantes de cada conglomerado en las dimensiones y subdimensiones de la escala.

El conglomerado 1 (tutor con dedicación alta) está conformado por 36 por ciento de la muestra de tutores. Se caracteriza por presentar un nivel alto en todos los componentes que miden el funcionamiento de la acción tutorial, así como en el desempeño de su labor como tutor. No obstante, podemos observar puntuaciones más bajas a la hora de conectar la realidad con la acción tutorial (ítem 17), la disposición de tiempo para organizar las tutorías (ítem 47), el empleo de libros de tutorías elaborados por editoriales (ítem 79), el apoyo de la administración educativa (ítem 81) y la aplicación de encuestas para valorar la práctica tutorial (ítem 85).

El 41 por ciento de la muestra de tutores pertenece al conglomerado 2 (tutor con dedicación moderada). Estos tutores se caracterizan por mantener niveles medio altos en las dimensiones planificación (análisis de la realidad y organización de la acción tutorial) y actuaciones del tutor (alumno, familia y profesorado); niveles medios en la dimensión desarrollo de la acción tutorial (programas $\mathrm{y}$ actividades, acciones en el aula y recursos TIC); y bajos en la dimensión evaluación. Asimismo, observamos una serie de variables que presentan puntuaciones altas que corresponden a la dimensión desarrollo, vinculadas a la planificación y atención del alumnado, tales como el empleo de diferentes técnicas y estrategias para el desarrollo del pensamiento crítico (ítem 50), el uso de estrategias de aprendizaje y técnicas de estudio (ítem 57), la concienciación del alumnado sobre la igualdad de género (ítem 58), el registro de la información durante las reuniones, entrevistas y actividades (ítem 69), y la disposición de material necesario para cumplir con la tutoría: listas, teléfonos... (ítem 70).

Por último, los docentes pertenecientes al conglomerado 3 (tutor con dedicación leve) está configurado por 23 por ciento de la muestra. Se caracteriza por presentar un nivel bajo en las dimensiones desarrollo (programas y actividades, acciones desde el aula y recursos TIC) y evaluación de la acción tutorial; medio en la planificación de la acción tutorial (análisis de la realidad y organización de la acción tutorial); medio alto en las actuaciones del tutor y más altas aquéllas centradas en el alumnado.

\section{Percepciones de los estudiantes}

En cuanto a los resultados sobre los perfiles de tutores por parte de los estudiantes, se siguió el mismo método de análisis (K-medias), y se fijó inicialmente el número de cluster en 6 conglomerados - debido a la magnitud de la muestra y la heterogeneidad de las respuestas- con el fin de evaluar el comportamiento de cada una de las variables del estudio. Los resultados de este primer análisis cluster mostraron los siguientes datos: se observó que apenas existían diferencias entre los conglomerados 1 y 6 y los conglomerados 4 y 5 . Ante estos resultados, se procedió a continuar el análisis cluster modificando el número de conglomerados a 5. Se tuvieron en cuenta los datos de dos conglomerados ( 3 y 4 ) en los que apenas existían diferencias entre las variables, por lo que se decidió modificar el número de conglomerados a 4. De este modo, al realizar de nuevo el análisis se obtuvieron resultados 
adecuados y de sencilla y significativa interpretación. En la Tabla 3 se puede apreciar el número de conglomerados finales y las medias de las respuestas de cada ítem.

Tabla 3. Centro de conglomerados finales (estudiantes)

Método K-medias. Solución 4 conglomerados

\begin{tabular}{|c|c|c|c|c|}
\hline Ítems & & ongl & herad & \\
\hline Funciones con el alumnado & 1 & 4 & 3 & 2 \\
\hline 1. El tutor está preparado para resolver las dudas que le planteamos los alumnos & 4.27 & 3.86 & 4.67 & 2.83 \\
\hline 2. El tutor habla con nosotros, aunque no lo pidamos & 3.75 & 3.43 & 4.35 & 2.45 \\
\hline 3. El tutor nos sugiere actividades de refuerzo si nos hacen falta & 3.68 & 3.26 & 4.30 & 2.21 \\
\hline 4. El tutor se preocupa por nuestros problemas personales & 3.69 & 3.25 & 4.46 & 2.06 \\
\hline 5. El tutor habla con la clase cuando surgen problemas & 4.46 & 4.21 & 4.80 & 3.07 \\
\hline 6. El tutor contribuye a solucionar los problemas de la clase & 4.27 & 3.85 & 4.66 & 2.66 \\
\hline 7. El tutor sabe tus aficiones, gustos y temas de interés & 2.74 & 2.25 & 3.31 & 1.66 \\
\hline 8. Elaboramos las normas de clase con el grupo y el tutor & 2.95 & 2.34 & 3.74 & 1.66 \\
\hline 9. El tutor nos ayuda a relacionarnos con los compañeros & 3.17 & 2.56 & 4.06 & 1.67 \\
\hline 10. En las tutorías nos ayudan a sentirnos bien trabajando en equipo & 3.34 & 2.49 & 4.17 & 1.60 \\
\hline 11. En las tutorías me enseñan técnicas de estudio que me ayuden a aprender mejor & 3.20 & 2.37 & 3.97 & 1.78 \\
\hline 12. El tutor me motiva para aprender & 3.62 & 2.84 & 4.46 & 1.89 \\
\hline Funciones con las familias & & & & \\
\hline $\begin{array}{l}\text { 13. Mis padres conocen las actividades diarias del colegio a través de los recursos } \\
\text { tecnológicos }\end{array}$ & 3.28 & 2.80 & 3.71 & 2.28 \\
\hline 14. El tutor habla con mis padres cuando tengo problemas & 3.61 & 3.29 & 4.28 & 2.32 \\
\hline 15. Los tutores informan a mis padres sobre lo que hago en clase & 3.48 & 3.22 & 4.11 & 2.51 \\
\hline Desarrollo de las tutorías & & & & \\
\hline $\begin{array}{l}\text { 16. En las tutorías se llevan a cabo debates sobre temas de actualidad que me ayudan a } \\
\text { pensar sobre los valores de la vida }\end{array}$ & 3.27 & 2.52 & 4.10 & 1.79 \\
\hline 17. El tutor nos informa sobre temas de nuestro interés (ocio, salud, sexualidad) & 2.93 & 2.22 & 3.76 & 1.54 \\
\hline 18. El tutor me ayuda a saber lo que es bueno para mí & 3.60 & 2.93 & 4.43 & 1.88 \\
\hline 19. Las tutorías me ayudan a decidir sobre mi futuro académico... & 3.02 & 2.32 & 3.85 & 1.72 \\
\hline 20. Las tutorías me ayudan a decidir sobre mi futuro profesional... & 2.83 & 2.11 & 3.72 & 1.56 \\
\hline Evaluación de las tutorías & & & & \\
\hline 21. En las tutorías utilizamos dinámicas distintas a otras asignaturas... & 3.09 & 2.39 & 3.63 & 1.81 \\
\hline 22. Estoy interesado por aquellos temas que trabajamos en las tutorías & 3.45 & 2.72 & 4.06 & 2.06 \\
\hline 23. Me siento cómodo con las actividades que realizamos en las tutorías & 3.79 & 3.09 & 4.43 & 2.29 \\
\hline 24. Opinamos sobre las actividades realizadas en las tutorías & 3.20 & 2.47 & 3.96 & 1.82 \\
\hline 25. Estoy satisfecho con el funcionamiento de las tutorías & 3.70 & 2.85 & 4.45 & 1.93 \\
\hline 26. Pienso en el trabajo que realizo en las tutorías & 2.90 & 2.01 & 3.84 & 1.54 \\
\hline 27. Valoro las tutorías que se realizan en clase & 3.47 & 2.66 & 4.29 & 1.89 \\
\hline 28. Las tutorías me ayudan a mejorar como persona & 3.32 & 2.28 & 4.25 & 1.60 \\
\hline 29. Pongo en práctica lo que aprendo en las tutorías & 3.05 & 2.12 & 3.92 & 1.62 \\
\hline
\end{tabular}

Fuente: elaboración propia. 
Tras las modificaciones, se obtuvo una solución óptima de cuatro perfiles: conglomerado 3: tutor con dedicación alta; conglomerado 1: tutor con dedicación moderada; conglomerado 4: tutor con dedicación leve; y conglomerado 2: tutor sin dedicación.

Se llevó a cabo el análisis de varianza (Hair et al., 2009) a través de la prueba de Levene, indicada anteriormente. Los estudios posteriores presentaron diferencias significativas entre los grupos en todas las dimensiones, que cumplen con los siguientes criterios: el conglomerado 3 presenta puntuaciones más altas con respecto al resto de grupos, siendo más moderadas en el conglomerado 1 , mientras que éste presenta medias superiores con respecto a los conglomerados 4 y 2. Por último, el conglomerado 2 presenta medias más bajas respecto del resto de los grupos. A continuación, mostramos los resultados y destacamos las variables que presentan mayores diferencias significativas.

El conglomerado 3 (tutor con dedicación alta) está conformado por 23 por ciento de la muestra de estudiantes. En este grupo el tutor presenta un alto grado de compromiso en sus funciones tutoriales respecto del alumnado, la familia, el desarrollo de actividades y en la evaluación de todo el proceso de las tutorías. Se obtienen mayores diferencias significativas en aquellas variables que se centran en la mediación en el aula (ítem 5), la resolución de problemas (ítem 6), así como aquellas actividades que promueven la participación en clase (ítem 8), las habilidades sociales (ítem 9), el trabajo en equipo (ítem 10), las técnicas de estudio (ítem 11), la motivación del alumnado (ítem 12), el interés de conocer de manera personal al alumnado (ítem 7) y aquellas acciones centradas en la comunicación e información con las familias (ítems 14 y 15).

El conglomerado 1 (tutor con dedicación moderada), con 35 por ciento de la muestra de estudiantes, se caracteriza por presentar niveles medios en todas las dimensiones que miden la tutoría y no presenta valores que puedan ser preocupantes para la labor tutorial. Este conglomerado mostró tener diferencias significativas en todas sus variables, especialmente puntuaciones más altas en la orientación individual del alumnado (ítem 1) y aquéllas que corresponden a la dimensión funciones del tutor con las familias. Por el contrario, las variables relacionadas con el conocimiento del alumnado (ítem 7), la participación en clase (ítem 8), el trabajo de las habilidades sociales (ítem 10), la toma de decisiones (ítems 26 y 27), el empleo de diferentes dinámicas (ítem 29), la reflexión (ítem 34) y transferencia de lo realizado en las tutorías (ítem 29) presentan puntuaciones más bajas.

El 28 por ciento de los estudiantes corresponde al conglomerado 4 (tutor con dedicación leve); presenta niveles bajos en el desarrollo de las tutorías y en los aspectos centrados en su evaluación, aunque mejoran levemente en las funciones con la familia y con el alumnado; destacan las variables que propician un buen clima en el aula (atención individualizada, resolución de problemas, mediación) y pasan más desapercibidas las variables que recogen competencias individuales del alumnado en su formación académica (habilidades sociales, participación, conocimiento del alumno, trabajo en equipo, técnicas de estudio y motivación).

Por último, el conglomerado 2 (tutor sin dedicación) está conformado por 14 por ciento de la muestra de estudiantes. Este grupo puntúa de manera baja en todas las variables, y la labor tutorial llega a ser escasa en los centros educativos; la variable centrada en los momentos que el tutor habla con el grupoclase cuando ocurre algún problema (ítem 5), presenta mayor puntuación.

\section{Síntesis de las muestras \\ de tutores y estudiantes}

En resumen, en las Gráficas 1 y 2 podemos observar tres perfiles de tutores que se asemejan en sus resultados. Encontramos docentes comprometidos en sus funciones como tutor, tutores con un compromiso más intermitente, pero que se mantienen en sus tareas, y otros que 
centran sus esfuerzos únicamente en mantener un clima de aula adecuado. Por último, se establece un cuarto perfil obtenido de la muestra de estudiantes denominado "tutor sin dedicación", que son aquéllos que no destacan en ninguna de las funciones tutoriales estudiadas.

\section{Gráfica 1. Medias en cada una de las dimensiones por conglomerados (profesorado)}

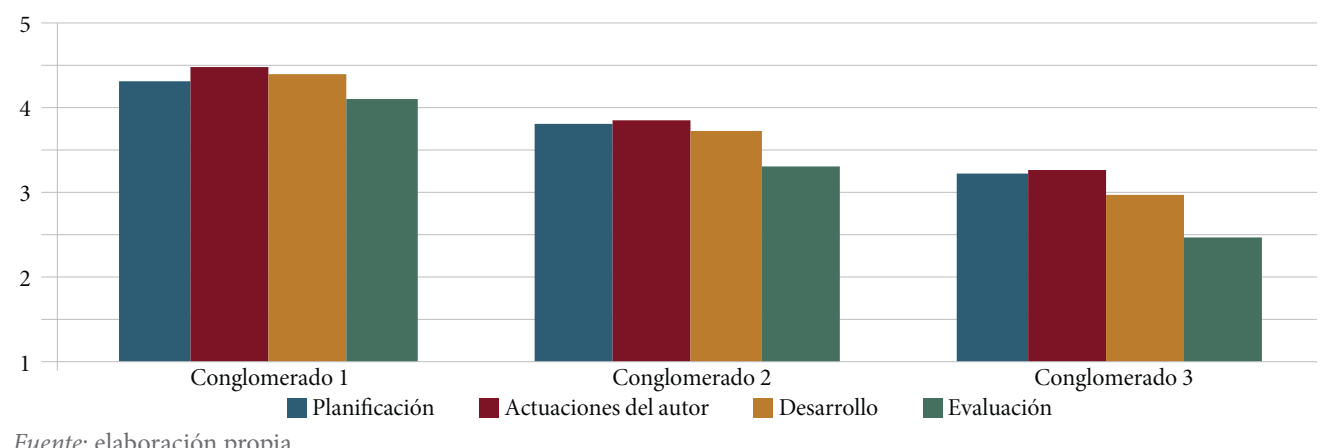

Gráfica 2. Medias en cada una de las dimensiones por conglomerados (estudiantes)

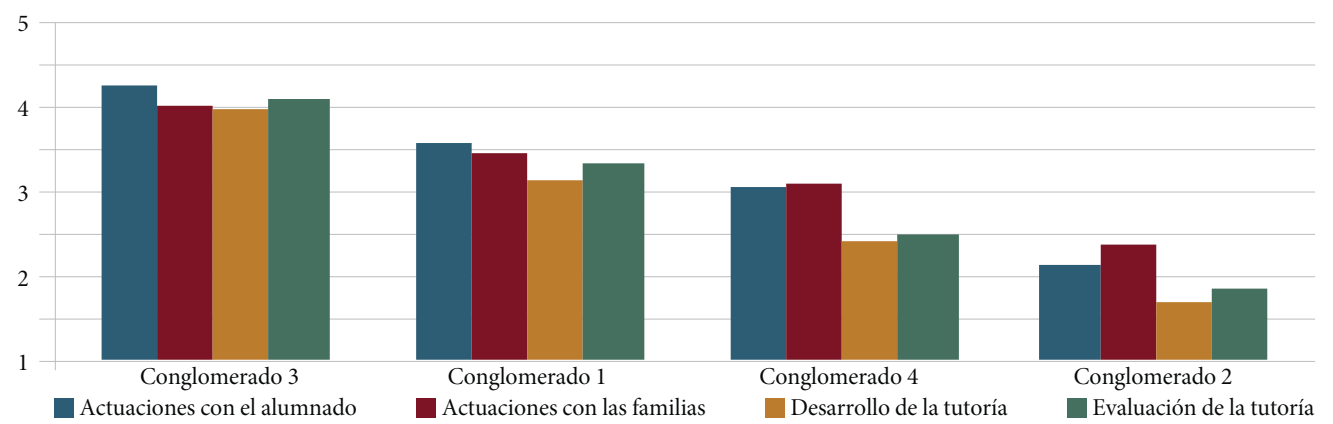

Fuente: elaboración propia.

\section{DisCUSIÓN Y CONCLUSIONES}

A la luz de los datos obtenidos se pudieron identificar posibles perfiles del tutor en los centros educativos de la Comunidad de Madrid, en función de su desempeño, tras la aplicación de dos análisis cluster mediante el agrupamiento y la distribución de las respuestas de los participantes (profesores-tutores y estudiantes). Los resultados obtenidos permitieron definir tres perfiles de profesores tutores, atendiendo a sus autovaloraciones, mientras que, con relación a las valoraciones de los estudiantes, se definieron cuatro perfiles. A continuación, se muestran las conclusiones procedentes de los resultados de ambos participantes y se destacan las coincidencias en los siguientes tres perfiles de tutores:
Tutores con dedicación alta: se trata de docentes con un alto grado de compromiso en sus funciones tutoriales y en todos los procesos implicados en el funcionamiento de la acción tutorial: planificación, desarrollo y evaluación. Tal y como expresan Kara y Can (2019), este tipo de docentes da respuesta al desarrollo integral de los estudiantes y muestra un alto compromiso con su labor. Este perfil agrupa tutores que realizan análisis de la realidad para adecuar la planificación de las tutorías a las necesidades de la comunidad educativa, y que establecen las líneas y ámbitos de intervención según la prioridad y el grado de necesidad (Arnaiz et al., 2015).

En cuanto a sus cualidades, destaca que estos tutores son: cercanos, preocupados, resolutivos, atentos, comunicativos y motivadores. De igual modo, conocen bien a cada estudiante, 
contribuyen en dar respuesta a sus necesidades y promueven el trabajo en equipo, el establecimiento y reparto de tareas y la potenciación de hábitos de autonomía personal y social (Youde, 2016; Waltz, 2019; Delgado-García et al., 2020). En cuanto al trato con las familias, son informativos, mediadores y orientadores. Por otro lado, mantienen un alto grado de colaboración con el resto de los profesores, centrada en dar respuesta a las necesidades del alumnado (Urosa y Lázaro, 2017).

En la práctica de la tutoría predomina el desarrollo de actividades que fomentan las interacciones en el grupo a través de debates sobre temas de actualidad, así como el empleo de técnicas para el desarrollo del pensamiento crítico y para llevar estilos de vida saludable; aportan orientación y apoyo al aprendizaje, mejora de la capacidad de aprender y de pensar, estrategias de aprendizaje y uso de recursos interactivos y acciones específicas para la mejora continua (registros de la información, listados e impresos, entre otros) (Castellano y Pantoja 2017; Martínez et al., 2019; Yale, 2019; Delgado-García et al., 2020). Por último, los tutores hacen seguimiento de la acción tutorial y actualizan el plan de acción tutorial (PAT) durante el curso, así como el contenido de las tutorías, además de realizar una evaluación final donde se establecen los objetivos de trabajo para el próximo año y la propuesta de acciones de mejora a partir de la detección de los puntos fuertes y débiles (Ponce et al., 2020).

Tutores con dedicación moderada: se trata de docentes que no descuidan sus funciones tutoriales ni aquellos aspectos centrados en la planificación y desarrollo de la acción tutorial, pero que sí muestran niveles más bajos a la hora de establecer criterios de evaluación. Este último aspecto debe estar estrechamente vinculado con los diferentes componentes de la acción tutorial para hacer posible la mejora educativa; la evaluación es un elemento que se debe establecer con la finalidad de obtener herramientas que conecten la función tutorial y la función docente (Bereziartua et al., 2017; Sa et al., 2019).

Los resultados muestran a tutores con un alto grado de compromiso en la planificación de las tutorías, en coherencia con la documentación organizativa de los centros educativos. Son fieles a las líneas de actuación marcadas en el PAT, además de considerar prioritario el desarrollo de la labor tutorial (Torquemada y Jardínez, 2019).

En cuanto a sus funciones tutoriales, destacala promoción del trabajo en equipo para favorecer la comunicación y la cohesión del grupo y el desarrollo de la autonomía individual y social de los estudiantes. Con las familias destaca su intervención cuando los estudiantes presentan algún tipo de problema durante el curso, ya que proporcionan información y orientación (Álvarez-Justel, 2017). Del mismo modo, los tutores cuentan con el resto de los profesores a la hora de analizar las dificultades de los alumnos para abordarlas conjuntamente.

En el desarrollo de las tutorías, emplean diferentes técnicas y estrategias para el desarrollo del pensamiento crítico y proporcionan una orientación individualizada ajustada a las necesidades de los estudiantes (Bejar, 2018). En cuanto a las características asociadas a la evaluación, se mantienen valoraciones más altas en la actualización de los contenidos trabajados en las tutorías, así como en la modificación del PAT durante el curso.

Tutores con dedicación leve: se trata de docentes que se preocupan en mantener sus funciones tutoriales, sobre todo aquéllas centradas en los estudiantes y en el desarrollo de un buen clima en las relaciones entre ellos. Sin embargo, los aspectos principales sobre el funcionamiento de la acción tutorial tienen valoraciones muy bajas (planificación, desarrollo y evaluación de la acción tutorial). En este sentido, Jiménez y Navaridas (2012) y Carvalho y Taveira (2014) coinciden en la importancia de un docente implicado en la atención de la diversidad de sus estudiantes y 
las relaciones que se establecen en el aula, pero sin descuidar los otros aspectos que contribuyen al funcionamiento de la acción tutorial.

Entre sus características destaca que elaboran el PAT acorde con la documentación del centro educativo: más de la mitad de los que se agrupan en este perfil se sienten implicados de manera activa en la planificación de la acción tutorial. En cuanto a sus funciones con el alumnado, lo valoran positivamente, sobre todo en lo que respecta a la resolución de problemas, la mediación de conflictos en el aula y la orientación personalizada (Cruz, 2018). Con respecto a la atención a las familias, ésta se realiza mediante el establecimiento de reuniones informativas. Al igual que con el profesorado, la principal característica es el seguimiento de los estudiantes que presentan mayores dificultades, mientras que, en el desarrollo y la evaluación de la acción tutorial, los tutores mantienen valoraciones bajas en la mayoría de sus indicadores.

Por último, los estudiantes identificaron un perfil más: tutores sin dedicación. Se trata de docentes con valores muy bajos en todas las áreas de la tutoría, de manera que la labor tutorial en esos centros educativos es preocupante. El hecho de que la identificación de este perfil haya sido posible únicamente con las valoraciones de los estudiantes puede reflejar un posible nivel de autocomplacencia por parte de los docentes, pues ninguno se considera a sí mismo un tutor que no desempeñe de forma adecuada su acción tutorial. Dado lo anterior, resulta fundamental para la comunidad educativa prestar especial atención a las diferencias encontradas, ya que podrían servir de base para una profunda reflexión a nivel interno. Se debe intentar superar los obstáculos y barreras que se exponen en la literatura (escasez de tiempo, falta de horario, diversidad de los estudiantes, falta de colaboración de las familias, insuficiente formación en orientación familiar y en acción tutorial, falta de organización de las tutorías) y considerar la importancia de aspectos como el respeto a los ritmos, la atención a las relaciones centro-contexto social, y la implicación activa del alumnado en su propio aprendizaje, entre otras (Arnaiz et al., 2015), además de valorar alternativas que posibiliten un buen funcionamiento de la acción tutorial.

Los resultados de este estudio expresan la necesidad de organizar las tutorías siguiendo un modelo sistemático que recoja los diferentes aspectos que intervienen en el funcionamiento de la acción tutorial; como dice Sanz (2009), el establecimiento de diversos criterios que permitan conocer, hacer y responder a las diferentes acciones desde la comunidad educativa.

Como la literatura científica expresa, el tutor debe contar con una serie de cualidades y competencias que le permitan desempeñar su función docente y tutorial (García Nieto, 2011; Youde, 2016). Aunque ya desde su definición no hay un consenso común (González-Benito y Vélaz de Medrano, 2014), a raíz del estudio se pueden señalar aspectos que es necesario reforzar para definir áreas de mejora en el trabajo diario de cada centro: la conexión de las tutorías con la realidad social, la dedicación de mayor tiempo a la organización de las tutorías, el empleo de recursos interactivos y didácticos para una mayor diversidad de métodos de enseñanza y aprendizaje, la atención de las necesidades educativas de los estudiantes, la promoción de programas y actividades que fomenten el desarrollo integral de los estudiantes, la reflexión y transferencia de lo aprendido y la valoración de la práctica tutorial, tanto por parte del estudiante como por el tutor.

Ante los resultados obtenidos y las conclusiones reseñadas se puede concluir que el estudio aporta información válida y relevante a la comunidad científica para seguir avanzando en el campo de estudio de la acción tutorial. Asimismo, el estudio permite establecer estrategias y herramientas que mejoren el trabajo de los tutores respecto a las acciones definidas en el estudio y las características propias de cada perfil; sin embargo, no está 
exento de limitaciones, por lo que se hace necesario plantear algunas cuestiones.

En primer lugar, el inevitable factor de autocomplacencia que destaca en los resultados del profesorado, el cual puede haber influido en las elevadas valoraciones que ellos hacen de su trabajo; es decir, puede ser que los tutores tiendan a relegar la autocrítica. Por esta razón, contrastar su percepción con la de los estudiantes resulta especialmente útil.

En segundo lugar, es importante que la evaluación de la acción tutorial esté más presente, ya que, como se observa en los resultados, constituye una tarea pendiente. Esto puede deberse a la concepción que tanto el profesorado como

\section{REFERENCIAS}

Alegre, Olga María, Remedios Guzmán y Carmen N. Arvelo (2017), "La tutoría y la inclusión en la formación del profesorado de educación primaria”, Educatio Siglo XXI, vol. 35, núm. 2, pp. 43-64.

Álvarez-Justel, Josefina (2017), "La tutoría en secundaria”, Educatio Siglo XXI, vol. 35, núm. 2, pp. 65-90.

Arnaiz, Pilar, Remedios de Haro y José-Manuel Guirao (2015), “La evaluación en educación primaria como punto de partida para el desarrollo de planes de mejora inclusivos en la Región de Murcia”, Revista Electrónica Interuniversitaria de Formación del Profesorado, vol. 18, núm. 1, pp. 103-122.

BeJAR, Luis Humberto (2018), "La tutoría como instrumento esencial para el desarrollo personal, comunitario, social y el aprendizaje intelectual de los/as estudiantes de nivel superior, de manera integrada e integradora a la vida", Revista Universidad y Sociedad, vol. 10, núm. 1, pp. 52-58.

Bereziartua, Justo, Arkait Zubiri, Nahia Intxausti y Ainhoa Odriozola (2017), “Acción tutorial en la transición del alumnado de las escuelas del medio rural a la educación secundaria”, Tendencias Pedagógicas, vol. 29, pp. 189-190.

Cano González, Rufino, Santiago Castillo, Mónica Casado y Ana Ponce de León (2013), “Estructura y organización de la orientación educativa en el marco normativo actual", en Rufino Cano (coord.), Orientación y tutoría con el alumnado y las familias, Madrid, Biblioteca Nueva, pp. 59-110.

Carbonero, Miguel-Ángel, Juan-Antonio Valdivieso y Luis-Jorge Martín (2010), "Identificación de variables descriptivas del profesorado los estudiantes tienen de ella, es decir, que esté relegada institucionalmente a aspectos curriculares (aprobación o no de la asignatura), y no se le tenga en cuenta por su carácter formativo.

La investigación desarrollada llama la atención sobre la práctica tutorial que el profesorado realiza en los centros, y plantea la necesidad de profundizar en las diferentes acciones mediante la realización de otros estudios para que se puedan introducir mejoras en los procesos de planificación, desarrollo y evaluación del funcionamiento de la acción tutorial, así como en lo que concierne a la atención de las funciones del tutor con respecto a toda la comunidad educativa.

eficaz”, en José de Jesús Gázquez y María del Carmen Pérez Fuentes (coords.), La convivencia escolar. Aspectos psicológicos y educativos, Granada, GEU, pp. 395-402.

Carvalho, Marisa y María-do-Céu Taveira (2014), "El papel del profesorado en la toma de decisiones académico y profesionales del alumnado", Revista Española de Orientación y Psicopedagogía, vol. 25, núm. 3, pp. 20-35.

Castellano, Eufrasio Amador y Antonio Pantoja (2017), "Eficacia de un programa de intervención basado en el uso de las TIC en la tutoría", Revista de Investigación Educativa, vol. 35, núm. 1, pp. 215-233.

Cruz, Sara (2018), "Desarrollo de la tutoría en el Colegio de Ciencias y Humanidades", en Rosalía Orta (coord.), Hacia un modelo de intervención tutorial para el CCH, México, UNAM, pp. 15-38.

De lA Torre-Laso, Jesús (2019), "La retroalimentación evaluativa o feedback para los trabajos en grupo como estrategia de acción tutorial en la universidad", Revista Educación, vol. 43, núm. 1, pp. 1-12.

Delgado-García, Manuel, Sara Conde y Ángel Boza (2020), "Perfiles y funciones del tutor universitario y sus efectos sobre las necesidades tutoriales del alumnado", Revista Española de Pedagogía, vol. 78, núm. 275, pp. 119-143.

García Nieto, Narciso (2011), "La función tutorial en el ámbito educativo", Padres y Maestros, núm. 342, pp. 5-9.

Giner Tarrida, Antoni (2012), "Perfil competencial del tutor o de la tutora de enseñanza secundaria", Revista Española de Orientación y Psicopedagogía, vol. 23, núm. 2, pp. 22-41. 
GonzÁLez-Benito, Ana y Consuelo Vélaz de Medrano (2014), La acción tutorial en el sistema escolar, Madrid, UNED.

González-Lorente, Cristina y Natalia GonzálezMorga (2015), "Enseñar a transitar desde la educación primaria: el proyecto profesional y vital", Revista Electrónica Interuniversitaria de Formación del Profesorado, vol. 18, núm. 2, pp. 29-41.

HAIR, Joseph. F., William C. Black, Barry J. Babin y Rolph E. Anderson (2009), Multivariate Data Analysis, Upper Saddle River, Prentice Hall.

Jiménez, María-Asunción y Fermín Navaridas (2012), "Cómo son y qué hacen los 'maestros excelentes': la opinión de los estudiantes”, Revista Complutense de Educación, vol. 23, núm. 2, pp. 463-485.

KArA, Mehmet y Gülfidan Can (2019), “Master's Students' Perceptions and Expectations of Good Tutors and Advisors in Distance Education", International Review of Research in Open and Distributed Learning, vol. 20, núm. 2, pp. 1-19. DOI: https://doi.org/10.19173/irrodl. v20i2.3674

LEón-Carrascosa, Víctor (2018), El funcionamiento de la acción tutorial y factores de eficacia, Tesis Doctoral, Madrid, Universidad Complutense de Madrid.

León-Carrascosa, Víctor y María José FernándezDíaz (2019a), "Diseño y validación de una escala para evaluar el funcionamiento de las tutorías en educación secundaria”, Revista de Investigación Educativa (RIE), vol. 37, núm. 2, pp. 525-541. DOI: https://doi.org/10. 6018/rie.37.2.345251

LEÓN-Carrascosa, Víctor y María José FernándezDíaz (2019b), "Eficacia percibida en el desarrollo de las tutorías en educación secundaria”, Bordón. Revista de Pedagogía, vol. 71, núm. 2, pp. 109-123.

Ley Orgánica 8/2013 de 9 de diciembre para la Mejora de la Calidad Educativa (BOE núm. 295, 10 de diciembre), Madrid, Ministerio de Educación y Formación Profesional.

López, Inmaculada y Purificación González (2018), "La tutoría universitaria como espacio de relación personal. Un estudio de caso múltiple", Revista de Investigación Educativa, vol. 36, núm. 2, pp. 381-399.

Marcelo, Carlos y Carmen-Rocío Yot (2013), “Tareas y competencias del tutor online", Profesorado. Revista de Currículum y Formación del Profesorado, vol. 17, núm. 2, pp. 305-325.

Martínez Clares, Pilar, Francisco José Pérez Cusó y Natalia González Morga (2019), “QQué necesita el alumnado de la tutoría universitaria? Validación de un instrumento de medida a través de un análisis multivariante", Educación XX1, vol. 22, num. 1, pp. 189-213.

Martínez Clares, Pilar, Francisco Javier Pérez Cusó y Miriam Martínez Juárez (2018), “Aplicación de los modelos de gestión de calidad a la tutoría universitaria”, Revista Complutense de Educación, vol. 29, núm. 3, pp. 633-649.

Pérez, Francisco José, Cristina González, Natalia González y Mirian Martínez (2017), “Tutoría en la universidad: un estudio de caso en la Facultad de Educación de la Universidad de Murcia”, Educatio Siglo XXI, vol. 2, núm. 35, pp. 91-110.

Ponce, Salvador, Yaralin Aceves y Brenda Imelda Boroel (2020), "Exploración del comportamiento de un instrumento para evaluar la función de tutoría académica en el contexto de escuelas Normales", Revista Iberoamericana de Evaluación Educativa, vol. 13, núm. 1, pp. 139-155.

SA, Bidyadhar, Chidum Ezenwaka, Keerti Singh y Anwarul Azim Majumder (2019), “Tutor Assessment of PBL Process: Does tutor variability affect objectivity and reliability?", $B M C$ Medical Education, vol. 19, núm. 76, pp. 1-8. DOI: https://doi.org/10.1186/s12909-019-1508-z

SÁnchez-Porro, David Guillermo y Eva González Ortega (2017), "Los programas de mediación escolar como herramientas para la promoción de unas relaciones de pareja saludables en la adolescencia”, Revista Española de Orientación y Psicopedagogía, vol. 28, núm. 1, pp. 72-85.

SANZ, Rafael (2009), Tutoría y atención personal al estudiante en la universidad, Madrid, Síntesis.

Torquemada, Alma Delia y Lizbeth Jardínez (2019), "La formación de competencias docentes universitarias a partir de la evaluación del desempeño del tutor", Publicaciones, vol. 49, núm. 1, pp. 39-52.

Urosa, Belén y Santa Lázaro (2017), "La función tutorial en educación infantil y primaria: actividades que implica y dificultad percibida por el profesorado en su desarrollo", Educatio Siglo XXI, vol. 35, núm. 2, pp. 111-138.

Vélaz de Medrano, Consuelo, María-Asunción Manzanares, Esther López-Martín y Nuria Manzano-Soto (2013), "Competencias y formación de los orientadores escolares: estudio empírico en nueve comunidades autónomas", Revista de Educación, núm. extra, pp. 261-292.

Waltz, Scott B. (2019), “Tutor Training for Service Learning: Impact on self-efficacy beliefs", Mentoring \& Tutoring: Partnership in Learning, vol. 27, núm. 1, pp. 26-43.

Yale, Annabel T. (2019), “The Personal Tutor-Student Relationship: Student expectations and experiences of personal tutoring in higher education", Journal of Further and Higher Education, vol. 43, núm. 4, pp. 533-544.

Youde, Andrew (2016), “Tutor Emotional Competences Valued by Learners in a Blended Learning Context", European Journal of Open, Distance and e-learning, vol. 19, núm. 2, pp. 81-97. 